\title{
A Structure of Multi-flow Extrusion Mold
}

\author{
Zheng Jianli \\ Shanxi Northern xingan chemical \\ industries, ltd, Taiyuan, Shanxi 030008, \\ China
}

\author{
Bai Renhua \\ Beihang University, School of \\ automation science and electrical \\ engineering Beijing 100191, China \\ rewolfbrh@163.com
}

\author{
Gao Yuanlou \\ Beihang University, School of \\ automation science and electrical \\ engineering Beijing 100191, China \\ gaoyuanlou@263.net
}

\begin{abstract}
This paper research on the multi-flow structure of extrusion die, with Circumference uniformly distributed method that all flow channels are set in a same cycle. Combination with the production of certain explosive, Simulates the progress of production of explosive and Analysis the distribution of velocity parameters to judge the quality of the structure of mold. The result show that flow speed at the same position of different flow channel is tiny difference, with the small difference at different positions of same circumference within the same flow channel, it can generally meet the requirements of the production.
\end{abstract}

Key words-Extrusion die, speed distribution, simulation, analysis, Circumference uniformly distributed method

\section{INTRODUCTION}

Extrusion die is important equipment of forming of chemical drugs. The structure of mold has significant effect on the quality of explosive, especially for small-size explosive. In addition, taking into account the small size of explosive and productivity, extrusion die for small size of explosive always uses multi-stream channel structure which means it can product many productions of explosive once. Shunt device of multi-stream channel is key factor to decide quality of explosive, and circumference uniformly distributed method is to set the flow channel evenly arranged in a cycle. This paper researches on the impact of uniformly distributed shunt device on the quality of explosive based on a certain type of production in a chemical plant.

\section{MOLD STRUCTURE}

\section{A. Structure and Function.}

Extrusion die contains four parts. 1) The initial flow channel and its expansion, whose role is to change flow state of melt from the spiral to the straight forward, while the cone is divided into the hollow cylindrical melt. 2) Shunt stent zone (Zone 2), whose role is to shunt melt which comes from Zone 1 and make all of melt get into each flow channel, then explosive can be formed at the same time. The stent is the essential component to bear mandrel in the die center, and shunt tendons on stent can cut melt into different segments. 3) Compression zone (Zone 3), whose role is to eliminate the confluence seam caused by shunt tendons cut and non-parallel flow after compression, and avoid causing unsteady flow of melt. 4) Forming zone (Zone 4), where melt flow is expected to gradually flow from compression forced. Melt stress relax gradually, welding degree of melt confluence seam is further strengthened, and at same time internal stress caused by compressed is eliminated. This area is also known as end section port.

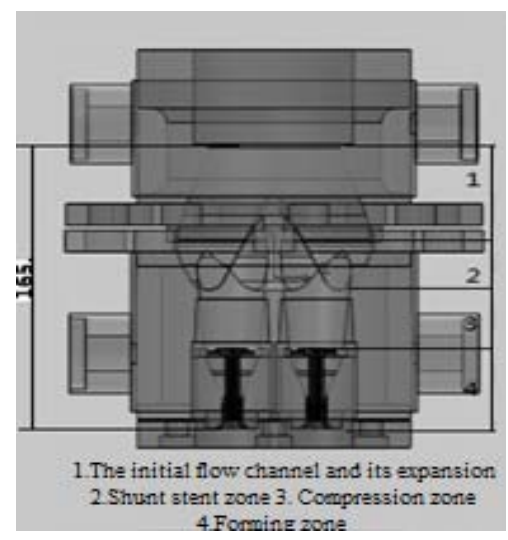

Fig1 Mold Zones

\section{B. Mold Important Parameters.}

The compression ratio is pressure ratio to describe plastic melt through extrusion die be cut into multiple units by shunt stent, conducting mutual welding pressure by squeezing. Compression ratio plays a decisive role in the formation of weld seam and strength of extrusion products. The melt strength of explosive is different from other plastic materials, there are more factors affected by the material. In order to make the products get more reliable weld strength, projects often use larger compression ratio.

$$
\varepsilon=\mathrm{a} / \mathrm{b}
$$

In the formula:

$\varepsilon$ - compression ratio

a -Maximum cross-sectional area of zone

b - export area

In this paper, the maximum cross-sectional area is $11309.7 \mathrm{~mm} 2$, and export area is $480.8 \mathrm{~mm} 2$, so compression ratio is 25.6 .

Length-diameter ratio is to describe the relationship of geometry of the extrusion products between orientation stereotypes in the stencil and the appropriate time. Length-diameter ratio is deeply related with the viscosity of materials. For large viscosity of materials, Ratio of length to diameter takes small value, and small viscosity large value. For explosive, length-diameter ratio is between 3 and 5 . 
$\mathrm{I}=\mathrm{L} / \mathrm{D}$

I- - Length-diameter ratio

L-C Length of forming zone

D-—Diameter of products

In this paper, forming length of mold is $45 \mathrm{~mm}$, there are 19 holes of $0.55 \mathrm{~mm}$ diameter in the center of export area, and the shape is $12.6 \mathrm{~mm}$ plum-shaped, Ratio of length to diameter is 3.5 .

\section{Mold Shunt Structure.}

Mold shunt structure is shown in Fig 2. Four flow channel holes of the shunt are uniformly distributed on the same circle, and at the center of each flow channel is vertebral body with the approximate taper with the initial flow expansion zone, whose role is to prevent fluid flow rate, so that four fluid velocities of flow channels are approximately the same. The periphery of the four flow channels is the cone of the $30^{\circ}$ taper, whose role is to smooth flow channel, increasing the flow rate of fluid nearby the flow channel. Between the flow channels is a saddle-shaped structure, which has smooth surface to not produce the phenomenon of fluid accumulation. The entire shunt flow channel is smooth, surface smooth and no dead ends. And the speed of shunt fluid in flow channel has small difference does not have significant differences.

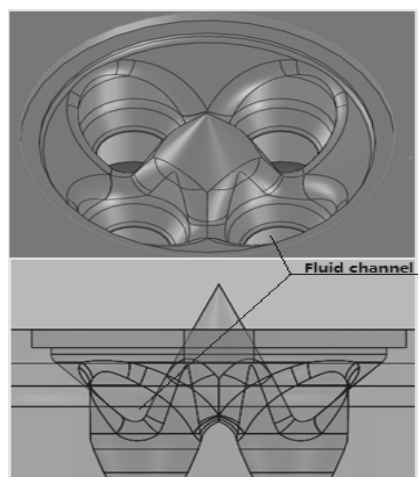

Fig 2 Forming segment

\section{Structure of forming.}

Forming export shape of the mold is cinquefoil, relative distance of the two sides is $12.6 \mathrm{~mm}$, and the largest distance of two points is $13.8 \mathrm{~mm}$. The mold has 19 holes of a diameter of $0.55 \mathrm{~mm}$. Forming structure is shown in Fig 3. Forming length is $45 \mathrm{~mm}$.

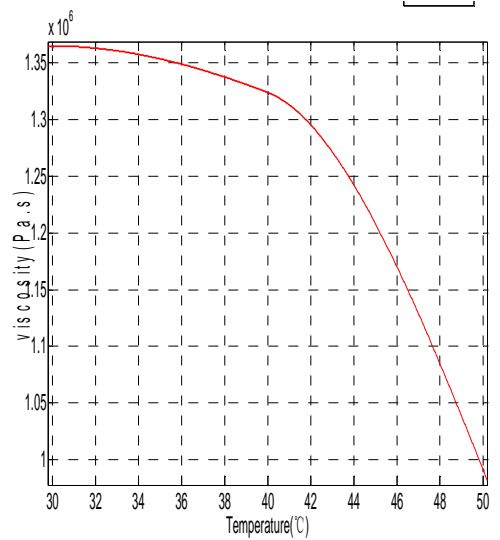

Fig 3 Temperature - viscosity curve

\section{SiMULATION AND ANALYSIS}

\section{A. Simulation Theory.}

Explosive melt is non-Newtonian fluid, and prior to molding, fluid follows the equation of the continuous fluid. Because of the mold in this simulation study with the columnar structure, the basic equation is written in the form of the stress components in cylindrical coordinates.

Continuity equation:

$$
\frac{\partial \rho}{\partial t}+\frac{1}{r} \frac{\partial\left(\rho r u_{r}\right)}{\partial r}+\frac{1}{r} \frac{\partial\left(\rho u_{q}\right)}{\partial \theta}+\frac{1}{r} \frac{\partial\left(\rho u_{z}\right)}{\partial z}=0
$$

In the formula:

$p=p(x, y, z, t)$ is the pressure equation, and the same as the follow.

$\rho=\rho(x, y, z, t)$ is density equation, and the same as the follow.

$u=u(x, y, z, t)$ is speed, the same as the follow.

Momentum equation:

$$
\rho\left(\frac{\partial u_{r}}{\partial t}+u_{r} \frac{\partial u_{r}}{\partial r}+\frac{u_{\theta}}{r} \frac{\partial u_{r}}{\partial \theta}+u_{z} \frac{\partial u_{z}}{\partial z}-\frac{u_{\theta}^{2}}{r}\right)=\rho f_{r}+\frac{1}{r}\left[\frac{\partial\left(r \tau_{r r}\right)}{\partial r}+\frac{\partial \tau_{\theta r}}{\partial \theta}+\frac{\partial\left(r \tau_{z r}\right)}{\partial z}\right]-\frac{\tau_{\theta \theta}}{r}
$$

In the formula:

$\tau_{i j}$ is the deviatoric stress tensor components, namely the relationship of stress and deformation rate in cylindrical coordinates.

$$
\begin{gathered}
\tau_{r r}=(-p+\lambda \nabla \cdot \vec{v})+2 \mu \frac{\partial u_{r}}{\partial r} \\
\tau_{\theta \theta}=(-p+\lambda \nabla \cdot \vec{v})+2 \mu\left(\frac{1}{r} \frac{\partial u_{\theta}}{\partial \theta}+\frac{u_{r}}{r}\right) \\
\tau_{z z}=(-p+\lambda \nabla \cdot \vec{v})+2 \mu \frac{\partial u_{z}}{\partial z}
\end{gathered}
$$

$$
\begin{gathered}
\tau_{r \theta}=\mu\left(\frac{\partial \mu_{\theta}}{\partial r}+\frac{1}{r} \frac{\partial u_{r}}{\partial \theta}-\frac{u_{\theta}}{r}\right) \\
\tau_{q z}=\mu\left(\frac{\partial u_{\theta}}{\partial z}+\frac{1}{r} \frac{\partial u_{z}}{\partial \theta}\right) \\
\partial_{z r}=\mu\left(\frac{\partial u_{r}}{\partial z}+\frac{\partial u_{z}}{\partial r}\right)
\end{gathered}
$$

In the formula, $\mu$ is viscosity.

Energy equation: 


$$
\begin{gathered}
\rho\left(\frac{\partial e}{\partial t}+u_{r} \frac{\partial e}{\partial r}+\frac{u_{\theta}}{r} \frac{\partial e}{\partial \theta}+u_{r} \frac{\partial e}{\partial z}\right)=\tau_{r r} \frac{\partial u_{r}}{\partial r}+\tau_{\theta \theta}\left(\frac{1}{r} \frac{\partial u_{\theta}}{\partial \theta}+\frac{u_{r}}{r}\right)+\tau_{z z} \frac{\partial u_{z}}{\partial z}+\tau_{r z}\left(\frac{\partial u_{z}}{\partial r}+\frac{\partial u_{r}}{\partial z}\right) \\
+\tau_{r \theta}\left(\frac{\partial u_{\theta}}{\partial r}+\frac{1}{r} \frac{\partial u_{r}}{\partial \theta}-\frac{u_{\theta}}{r}\right)+\tau_{\theta z}\left(\frac{1}{r} \frac{\partial u_{z}}{\partial \theta}+\frac{\partial u_{\theta}}{\partial z}\right)+\rho q+\frac{1}{r}\left[\frac{\partial}{\partial r}\left(k r \frac{\partial T}{\partial r}\right)+\frac{\partial}{\partial \theta}\left(k \frac{1}{r} \frac{\partial T}{\partial \theta}\right)+\frac{\partial}{\partial z}\left(k r \frac{\partial T}{\partial z}\right)\right]
\end{gathered}
$$

In the formula, $\mathrm{T}$ is temperature.

\section{B. Parameters of Explosive.}

The density of Explosive melt is $1710 \mathrm{Kg} / \mathrm{m}^{3}$, specific heat capacity is $1008 \mathrm{~J} /(\mathrm{kg} \mathrm{K})$, thermal conductivity of $0.14 \mathrm{~W}$ / M.K, and viscosity parameter shown in Fig 3.

\section{The boundary conditions.}

Inlet mass flow is $0.0008839 \mathrm{~kg} / \mathrm{s}$, environment pressure at output is 1 standard atmosphere (101325Pa), ambient temperature is $20{ }^{\circ} \mathrm{C}$, and forming export as the same with environment. Wall roughness is $0.4 \mu \mathrm{m}$, and the temperature of water in the water jacket is $30^{\circ} \mathrm{C}$.

\section{Analysis of Results.}

According to the mold structure and the boundary conditions and material conditions, use SolidWorks to simulate to obtain the velocity distribution of melt in mold which is shown in Fig 4, and compare curve of the flow channel at the same position to obtain results that are shown in Fig 5 and Fig 6. Fig 5 is result of four curves near the center of mold, and Fig 6 is result of four curves far away from the center of mold. Fig5 and Fig 6 show that four channels are same.

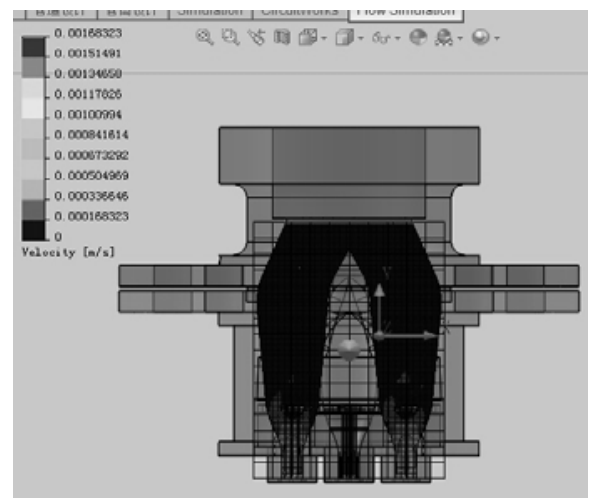

Fig4 Velocity Distribution

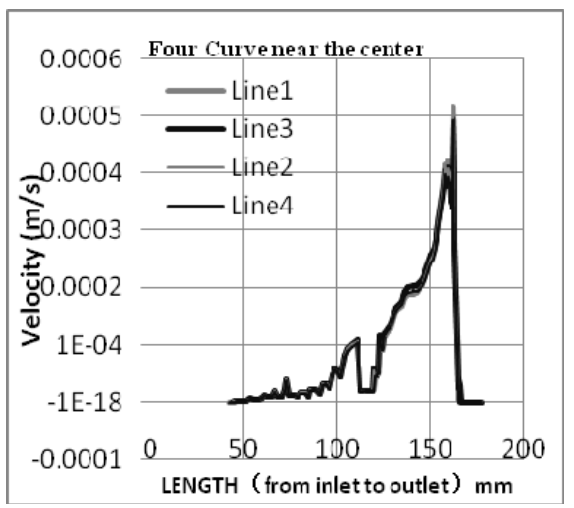

Fig 5 velocity distribution of the center

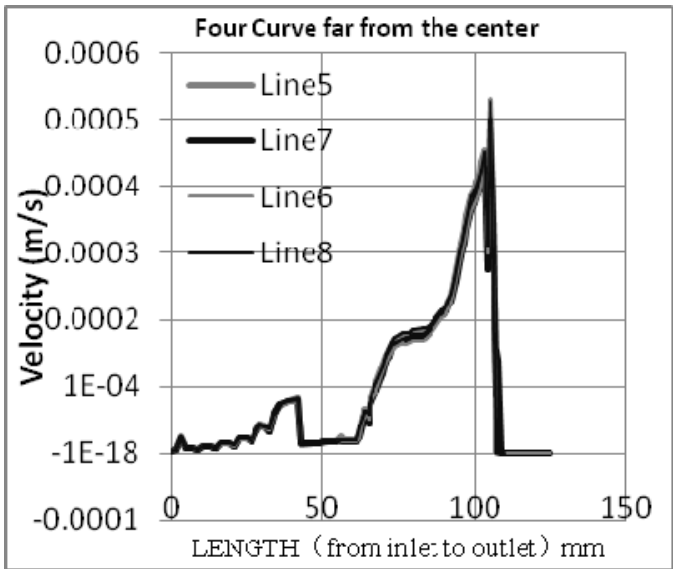

Fig 6 velocity distribution away the center

Analysis velocity fluctuation on the different place of same flow channel, the result is shown in Fig 7, length of forming zone is $45 \mathrm{~mm}$, velocity fluctuate at forming zone, in the forming zone, distance from outlet is smaller, fluctuate is smaller, the product is better.

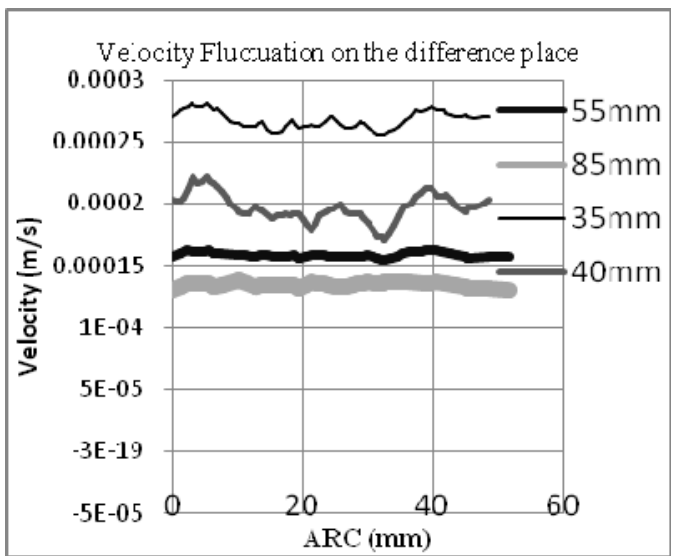

Fig 7 Velocity Fluctuation of a channel

\section{CONCLUSION}

In this paper, the designed mold meets the actual production requirements, while the circumference uniformly distributed triage mold is suitable for multi-flow channel forming. Since the circumference triage has an advantage in the design and processing, this mold form has a wide range of prospects in the relevant molding production.

\section{REFERENCES}

[1] Li Zhengguang PVC extrusion mold design, plastic, 2008 (3).

[2] Compilation group of the plastic mould design manual. Plastic injection mold design manual [M]. Beijing: China machine press, 1992. 